Rapid Communication

\title{
Uphill airflow transport of drops on superhydrophobic inclines
}

\author{
Dwayne Chung Kim Chung a , Mayur Katariya ${ }^{\text {a }}$, So Hung Huynh ${ }^{\text {a }}$, Brandon Huey-Ping Cheong ${ }^{\text {a }}$, Oi Wah Liew ${ }^{\text {, }}$, \\ Murat Muradoglu ${ }^{\mathrm{a}}$, Tuck Wah Ng ${ }^{\mathrm{a}}$ \\ a Laboratory for Optics \& Applied Mechanics, Department of Mechanical \& Aerospace Engineering, Monash University, Clayton VIC3800, Australia

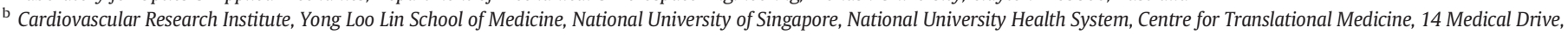 \\ Singapore 117599, Singapore
}

\section{A R T I C L E I N F O}

\section{Article history:}

Received 1 April 2015

Received in revised form 15 June 2015

Accepted 16 June 2015

Available online 5 August 2015

\section{Keywords:}

Superhydrophobic

Droplet

Liquid bridge

Airflow

Pinching

\begin{abstract}
A B S T R A C T
The ability of uphill droplet transport on a superhydrophobic surface incline using airflow was demonstrated. Analytical equations were found to describe the droplet volume at detachment at various inclination angles for the range of physical parameters applied. A pinching off behavior with necking prior to detachment was observed in which a constant time to rupture of $3.2 \times 10^{-2}$ s from a neck thickness of $1 \times 10^{-3} \mathrm{~m}$ was found regardless of the inclination angle used. The approach provided rapid uphill translation of 33 droplets per second and incurs limited aerosolization due to the small forces in action.
\end{abstract}

(c) 2015 Elsevier B.V. This is an open access article under the CC BY-NC-ND license (http://creativecommons.org/licenses/by-nc-nd/4.0/).
The ability of uphill droplet transport on a superhydrophobic surface incline using airflow was demonstrated. Analytical equations were found to describe the droplet volume at detachment at various inclination angles for the range of physical parameters applied. A pinching off behavior with necking prior to detachment was observed in which a constant time to rupture of $3.2 \times 10^{-2} \mathrm{~s}$ from a neck thickness of $1 \times 10^{-3} \mathrm{~m}$ was found regardless of the inclination angle used. The approach provided rapid uphill translation of 33 droplets per second and incurs limited aerosolization due to the small forces in action.

The capacity to transport liquid continuously uphill in a closed channel or pipe may seem rather unremarkable when one considers that a simple pump is able to do this. Yet, the ability to transfer individual droplets uphill has proven to be of great interest to the scientific community. This is increasingly significant due to the proliferation of open and discrete microfluidic systems [1,2]. The basis to transport droplets uphill was theoretically mooted almost 40 years ago [3] and then realized experimentally just over 20 years ago [4]. The modification of the wetting characteristics of surfaces in various ways provides an obvious approach to accomplish uphill droplet transport [4,5]. Nevertheless, the lateral vibration of surfaces [6], heating surfaces to attain the Leidenfrost effect [7], and illumination of laser beams on the substrate [8] or droplet [9] are among other methods that have also been advanced.

When droplets are placed on flat superhydrophobic (SH) surfaces they are able to assume an almost spherical shape when gravity is

E-mail address: engngtw@gmail.com (T.W. Ng). negligible and when Cassie wetting state is dominant [10]. These surfaces can now be fabricated using various ways $[11,12]$. They generally offer ultra-low friction which can present containment problems in applications unless suitable constraints are used $[13,14]$. Yet, the de-facto merit of using such surfaces lies with the low levels of sample loss due to the highly non-wetting characteristic offered, which makes it attractive for biochemical applications [13-16].

It has been recently shown that when liquid is delivered continuously through an orifice to a SH incline, the increasing weight of the drop will cause the rear contact angle to reduce to the extent where the drop eventually pinches off with the contact line to traverse over the orifice [17]. On making contact with the solid, however, a predominant Cassie wetting state is restored which will quickly allow the drop to detach and travel down the surface. This presents a viable means to rapidly generate droplets as opposed to other methods which can arguably provide more precise volumetric control [14]. In this work, we explore the use of airflow to engender the uphill transport of droplets forming from water delivered continuously through an orifice onto the inclined $\mathrm{SH}$ surface.

A T-shaped setup was fabricated (see Fig. 1) to conduct the experiments. As air was delivered along a straight passage from one opening to another diametrically opposite opening, it created a low pressure region at the intersection with the orthogonal passage, allowing air to be drawn in through the third opening. In the experiments, the airflow delivered was unchanged, such that the air velocity at the point of drop formation, measured using a pitot tube and digital manometer (Digitron, 2002), was kept at $24.9 \mathrm{~m} / \mathrm{s}$. The SH substrate was a copper plate with a small hole of $1 \mathrm{~mm}$ diameter drilled through, polished 


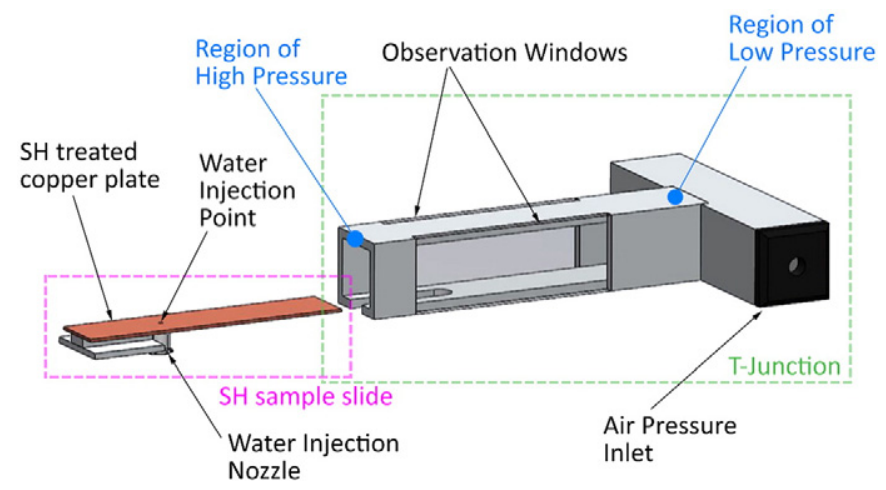

Fig. 1. The setup devised to obtain images of droplet transport. Water from a peristaltic pump was delivered through a pipe to an injection nozzle located at the bottom of a $\mathrm{SH}$ surface. This assembly is attached to the T-shaped setup, where air input into one arm to exit from the arm directly opposite to it, creates a low pressure region at the junction with the third arm. The higher (ambient) pressure at the inlet of the third arm then causes an airflow past the drop developing on the $\mathrm{SH}$ surface. Varied inclination measurements were obtained by tilting the entire setup. Images were obtained by placing the light source and camera at opposite sides of the transparent observation windows.

earlier to remove scratches using silicon carbide electro-coated waterproof abrasive paper (KMCA, WET/DRY S85 P600). Prior to use, it was first cleaned using absolute ethanol, allowed to air dry, and then immersed in a $24.75 \mathrm{mM}$ aqueous solution of silver nitrate $\left(\mathrm{AgNO}_{3}\right)$ for $1 \mathrm{~min}$ to form the micro- and nanostructures. After this, it was rinsed with copious amounts of distilled water followed by absolute ethanol before being allowed to air dry. Once dried, it was immersed in a $1 \mathrm{mM}$ solution of the surface modifier $\mathrm{CF}_{3}\left(\mathrm{CF}_{2}\right)_{7} \mathrm{CH}_{2} \mathrm{CH}_{2} \mathrm{SH}$ in absolute ethanol (ethanol with low water content) for $5 \mathrm{~min}$. After removal, it was again rinsed with copious amounts of distilled water, followed by absolute ethanol, and then air dried. This surface was characterized using a SEM (Helios NanoLab) and the contact angle behavior interrogated with video recording of a $10 \mu \mathrm{L}$ drop displaced using a $0.7176 \mathrm{~mm}$ outer diameter tip moving laterally on the DSA100S (Kruss). Distilled water was delivered via a flexible tube and adaptor located at the bottom of the plate through a programmable peristaltic pump (NE9000 New Era). The liquid flowrate was kept constant at $0.1 \mathrm{~mL} / \mathrm{s}$ to avoid the two body separation regime observed previously [17]. Videos of the drops forming on the SH surface were recorded using a high-speed camera (Fastec) at 250 frames per second.

A sample SEM image of the SH surface is given in Fig. 2(a). It can be seen that dendritic and granular structures are formed that allow a predominant Cassie wetting state to form. Fig. 2(b) furnishes a side view of the drop as it exhibits advancing and receding contact angles of $158.5^{\circ} \pm 0.1^{\circ}$ and $156.9^{\circ} \pm 0.18^{\circ}$ respectively (average and standard deviation taken from 260 images of the same drop displaced).

We first consider the case of drop formation and transport in the absence of airflow (see Fig. 3(a)-(c)). Due to continual liquid filling through the orifice, a liquid bridge first develops, from which it gradually constricts before rupturing. This makes it different from the case of tilting the surface for a drop without infilling where the advancing and receding contact angle hysteresis dictates its movement from the surface. Any drop movement in the lateral direction under the action of a lateral force coinciding with the surface being inclined at the limiting sliding angle will cause non-adherence to Amonton's law of friction [18]. Here, the liquid flowrate into the drop will vary the rupture resistance of the liquid bridge. Since the flowrate was kept unchanged, it is possible then to assume that a constant adhesion force $F_{\text {adh }}$ was in operation, and that the ability of the drop to be just detached from the surface is dictated by the balance of forces from gravity at equilibrium, such that

$$
V=\frac{F_{a d h}}{\rho_{w} g \sin \theta}=k_{1} \frac{1}{\sin \theta}
$$

where $V=$ volume, $\rho_{w}=$ the density of water, $g=$ gravitational acceleration, and $\theta=$ angle of inclination. The experimental trend of $V$ against $\theta$ is evident in Fig. 4 (scatter data), wherein a best fit process (solid line) yielded $k_{1}=0.018 \times 10^{-3} \mathrm{~m}^{3}$. Based on this, we have $F_{a d h}=1.76 \times 10^{-4} \mathrm{~N}$. This value is much larger than the adhesion forces of water drops on the same type $\mathrm{SH}$ surface previously found $\sim\left(10^{-9}\right)$ $\mathrm{N}$ [19], but is reasonable due to a different mechanism of attachment here (i.e. through a liquid bridge).

If we consider the case of drop formation and transport with airflow (see Fig. 3(d)-(f)), a somewhat similar case of a liquid bridge developing first, from which it gradually constricts before rupturing, can be seen. In being able to cause the droplet to detach and move uphill, the drag force developed has to overcome both the gravitational force and adhesion force of the drop to the surface. The drag force scales according to the cross sectional area $A$ of the drop if we assume the airflow velocity and drag coefficient (determined by its shape while interacting with the airflow) to be relatively unchanged. By conveniently equating to a sphere, it is possible to deduce that $A=4.836 \mathrm{~V}^{2 / 3}$. If we assume that the adhesion force to be unchanged in magnitude from the case without airflow but acting in the opposite direction, the force balance equation can be stated as

$$
4.836\left(\frac{1}{2} \rho_{a} u^{2} C_{d}\right) V^{2 / 3}-\rho_{w} g V \sin \theta-F_{a d h}=0
$$
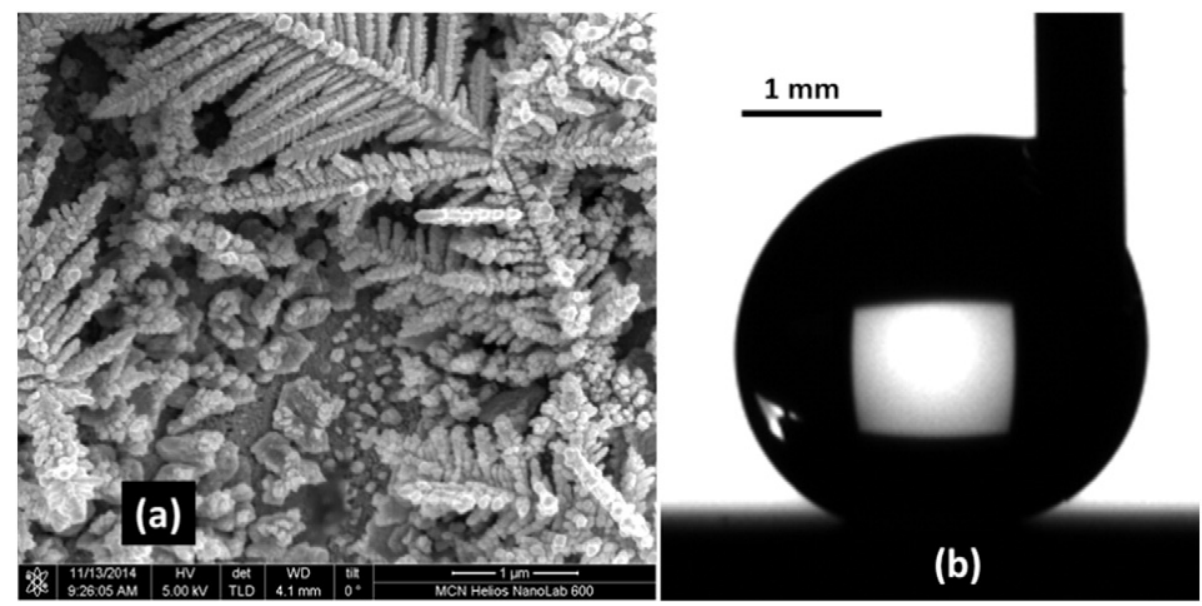

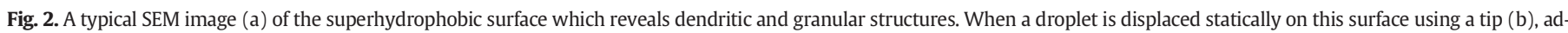
vancing and receding contact angles of $158.5^{\circ} \pm 0.1^{\circ}$ and $156.9^{\circ} \pm 0.18^{\circ}$ respectively were found. 

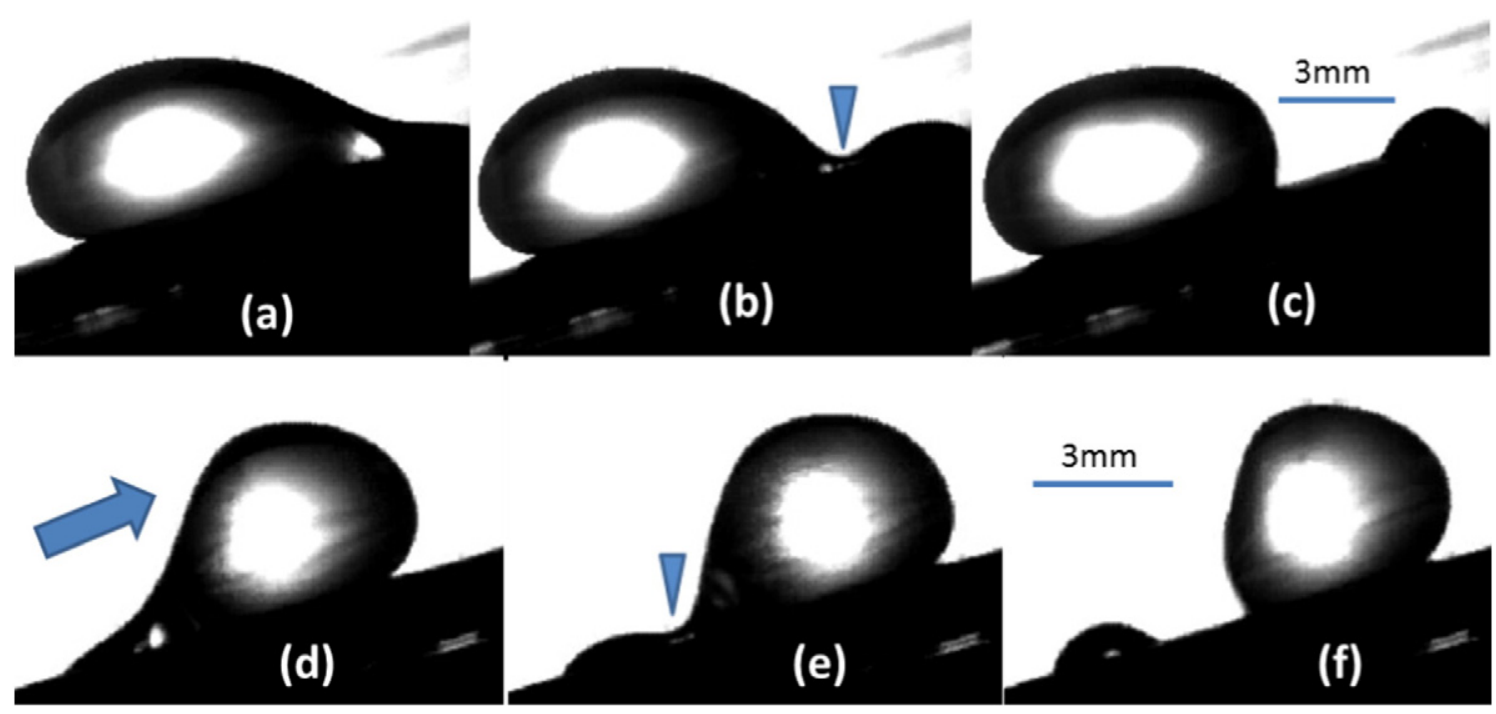

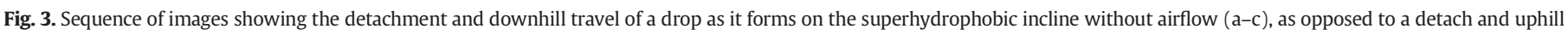

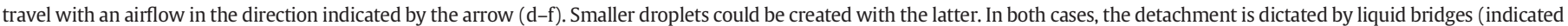
by arrows) formed which later constricted and ruptured.

where $\rho_{a}=$ the density of air, $u=$ airflow speed, and $C_{d}=$ drag coefficient. Eq. (2) can be rewritten, by grouping into constants, as

$k_{2} V^{2 / 3}-k_{3} V \sin \theta-k_{4}=0$.

On inspecting Eq. (3), one finds that it is a multivariable calculus problem, in which an implicit function theorem will allow relations to be converted to functions of several real variables. Essentially, there may not be a single function whose graph is the entire relation, but there may exist a solution based on restrictions placed on the domain of the relation. If we limit to $V>0$ and $\theta>0$, a theoretical fitting solution using Eq. (3) with $V$ scaled to $\mathrm{mL}$ from $\mathrm{m}^{3}$ is obtained with $k_{2}=0.23$, $k_{3}=1$, and $k_{4}=0.018$. While there is an absence of close conformance (which we believe is due to changes in $C_{d}$ during the evolution of the droplet as a soft matter rather than a solid), there is a consistent point at $\theta=18^{\circ}$ in which any increase in $\theta$ will not produce a solution in the domain. This is observed in the experiments. Based on fitting these values, one is able to determine that $C_{d}=0.012$. Using the airflow speed, air density, air viscosity, and the characteristic length (diameter of droplet) at detachment applied during the experiments, the Reynolds number is found to be 1020 . Based on this value, the $C_{d}$ for a sphere is calculated to be 0.023 using known relationships [20]. That the

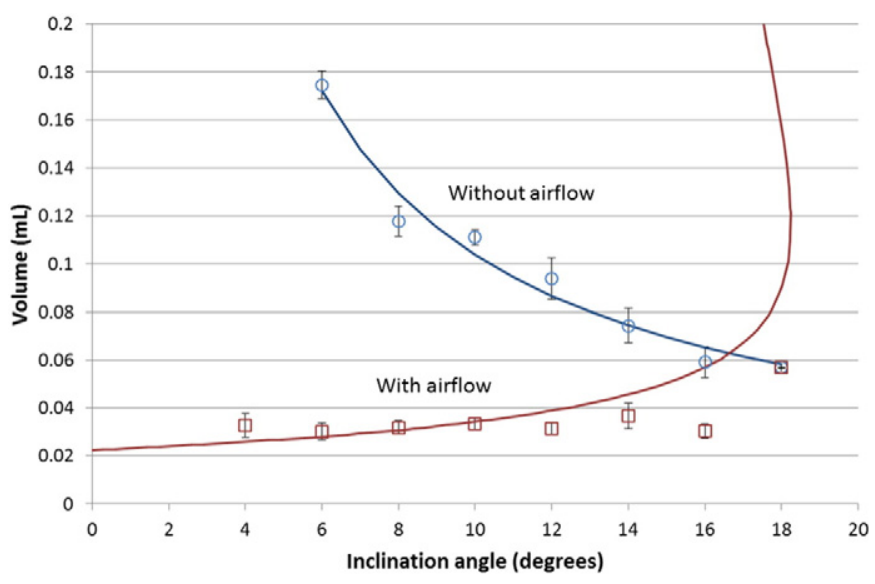

Fig. 4. Plots of drop volume developed with various incline angles of the SH surface with and without the airflow. The error bars indicate 1 standard deviation out of 5 readings made. The solid lines indicate best fit based on theory. experimental value is approximately half of the calculated value can be rationalized from the fact that the shape of the droplet (see Fig. 3(c)-(d)) is not fully spherical. It's more aerodynamic shape then accounts for the reduction in the drag coefficient. An interesting observation here is that smaller volume drops can be created and collected uphill when airflow is introduced. This has usefulness in microfluidic applications.

Since the droplet detachment mechanism (both uphill and downhill) is based on the pinching off effect, we examine the similarities and differences here with the case of pendant drops. With pendant drops, the liquid bridge elongates into a thin diameter $\sim O\left(10^{-4}\right) \mathrm{m}$ before it necks off quickly $\sim O\left(10^{-3}\right) \mathrm{s}$ to rupture when low in viscosity Newtonian fluids like water are used [21]. The experimental measurements with airflow here yielded a consistent time to rupture of $3.2 \times 10^{-2} \mathrm{~s}$ from a neck thickness of $1 \times 10^{-3} \mathrm{~m}$, notwithstanding the range of inclination angles used (see Fig. 5). This invariance confirms that the pinching off effect is capillary driven as in the case of the pendant drop. However, the approximately one order of magnitude higher in terms of the neck thickness and time to rupture implies contribution of the adhesion force of the drop to the surface which stabilizes and thus prolongs the necking to rupture process.

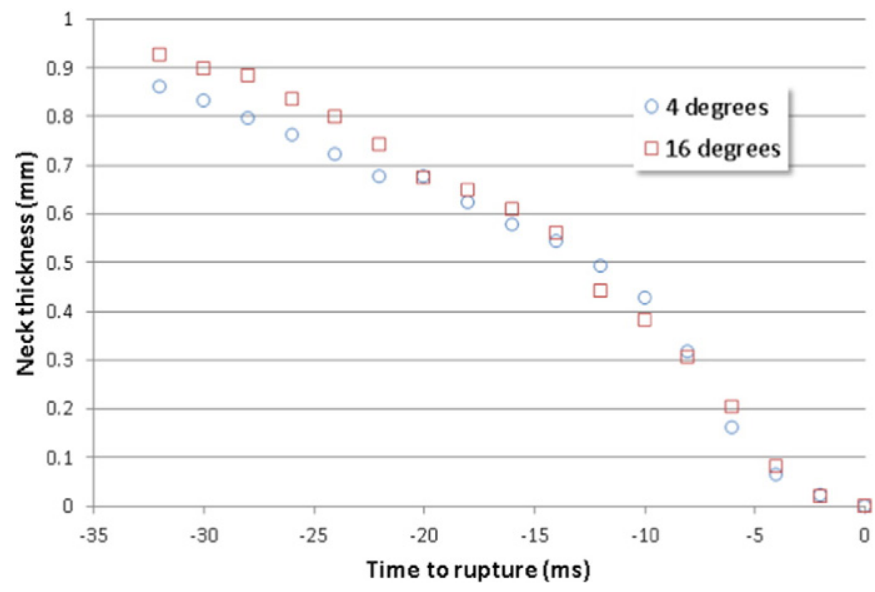

Fig. 5. Plots of the minimum thickness of liquid bridge's neck region at different times prior to rupture for inclination angles of $4^{\circ}$ and $16^{\circ}$ to the horizontal when airflow was applied. There is close correspondence in the trends. 
From an application perspective, the airflow approach here is useful because it allows uphill transport of a very rapid stream of droplets. Since water was delivered at a flowrate of $0.1 \mathrm{~mL} / \mathrm{s}$ and the average size of the droplets was $0.03 \mathrm{~mL}$, this translated to the delivery of 33 droplets per second. Due to the low forces involved $\sim O\left(10^{-4}\right) \mathrm{N}$, the approach should also produce lower sample losses through aerosolization, which is known to have significant negative implications if the sample were to contain pathogens such as bacteria [22].

In summary, we have shown the feasibility of uphill droplet transport using airflow on a superhydrophobic surface incline. The trend of volume on detachment in relation to inclination angle could be described using analytical equations for the range of physical parameters used in the experiment. A pinching off behavior with necking prior to detachment was observed, in which a constant time to rupture of $3.2 \times 10^{-2} \mathrm{~s}$ from a neck thickness of $1 \times 10^{-3} \mathrm{~m}$ was found regardless of the inclination angle.

\section{Acknowledgments}

T.W. and O.W. acknowledges funding support from the Australian Research Council Discovery Project DP120100583. This work was performed in part at the Melbourne Centre for Nanofabrication (MCN) in the Victorian Node of the Australian National Fabrication Facility (ANFF). Access to usage of the Kruss DSA100S system at the Institute of Frontier Materials, Deakin University is appreciated.

\section{References}

[1] M. Abdelgawad, A.R. Wheeler, Adv. Mater. 21 (2009) 920

[2] M.K. Chaudhury, A. Chakrabarti, S. Daniel, Langmuir (2015)http://dx.doi.org/10. 1021/la504925u.

[3] H.P.J. Greenspan, J. Fluid Mech. 84 (1978) 125.

[4] M.K. Chaudhury, G.M. Whitesides, Science 256 (1992) 1539.

[5] J. Zhang, Y. Han, Langmuir 23 (2007) 6136.

[6] P. Brunet, J. Eggers, R.D. Deegan, Phys. Rev. Lett. 99 (2007) 144501

[7] A. Grounds, R. Still, K. Takashina, Sci. Rep. 2 (2012) 720.

[8] A.Y. Vorobyev, C. Guo, Appl. Phys. Lett. 94 (2009) 224102.

[9] J. Berná, D.A. Leigh, M. Lubomska, S.M. Mendoza, E.M. Pérez, P. Rudolf, G. Teobaldi, F. Zerbetto, Nat. Mater. 4 (2005) 704

[10] G. Whyman, E. Bormashenko, J. Colloid Interface Sci. 331 (2009) 174.

[11] L. Wu, J. Zhang, B. Li, L. Fan, L. Li, A. Wang, J. Colloid Interface Sci. 432 (2014) 31.

[12] C.Y. Lau, T. Vuong, J. Wang, M. Muradoglu, O.W. Liew, T.W. Ng, Appl. Surf. Sci. 311 (2014) 89.

[13] T. Vuong, B.H.-P. Cheong, S.H. Huynh, M. Muradoglu, O.W. Liew, T.W. Ng, Lab Chip 15 (2015) 991.

[14] T. Vuong, A. Qi, M. Muradoglu, B.H.-P. Cheong, O.W. Liew, C.X. Ang, J. Fu, L. Yeo, J. Friend, T.W. Ng, Soft Matter 9 (2013) 3631.

[15] M. Zhou, J.H. Yang, X. Ye, A.R. Zheng, G. Li, P.F. Yang, Y. Zhu, L. Cai, J. Nano Res. 2 (2008) 129.

[16] J. Li, L. Li, X. Du, W. Feng, A. Welle, O. Trapp, M. Grunze, M. Hirtz, P.A. Levkin, Nano Lett. 15 (2015) 675.

[17] M. Katariya, T.W. Ng, J. Phys. D. Appl. Phys. 46 (2013) 345302.

[18] E. Bormashenko, Y. Bormashenko, G. Oleg, Langmuir 26 (2010) 12479.

[19] T.W. Ng, Y. Panduputra, Langmuir 28 (2012) 453.

[20] F.A. Morrison, An Introduction to Fluid Mechanics, Cambridge University Press, New York, 2013.

[21] J.R. Castrejón-Pita, A.A. Castrejón-Pita, E.J. Hinch, J.R. Lister, I.M. Hutchings, Phys. Rev. E 86 (2012) 015301.

[22] A.G. Turner, J.R. Wilkins, J.G. Craddock, J. Clin. Microbiol. 1 (1975) 289. 